\title{
Roentgenographic diagnosis of mucopolysac- charidosis with particular reference to Morquio syndrome
}

\author{
Umesh Parashari, MB BS, MD (Radiodiagnosis) \\ Sachin Khanduri, MB BS, MD (Radiodiagnosis) \\ Samarjit Bhadury, MB BS, MD (Radiology) \\ Sugandha Rawat, MB BS, DMRD (Std)
}

Department of Radiodiagnosis, Era's Lucknow Medical College, Lucknow, Uttar Pradesh, India

Corresponding author: U Parashari (drumesh.rd@gmail.com)

\begin{abstract}
Mucopolysaccharidosis (MPS) comprises a group of conditions associated with an abnormality in glycoprotein or mucopolysaccharides metabolism. Types of MPS identified are MPS I-H (Hurler's syndrome, gargoylism), MPS II (Hunter's syndrome), MPS III (Sanfilippo's syndrome), MPS IV (Morquio-Brailsford syndrome), MPS I-S (Scheie's syndrome) and MPS VI (MaroteauxLamy syndrome). The Hunter type is inherited as an X-linked recessive; the others are autosomal recessive. Patients with MPS IV can usually be clinically distinguished from patients with other forms of MPS; their intelligence is unimpaired, in contrast with other forms of MPS. Husler coined the term dysostosis multiplex to describe the skeletal findings.
\end{abstract}

\section{Introduction}

Mucopolysaccharidosis (MPS) is a rare inherited biochemical disorder characterised by the accumulation of mucopolysaccharides (glycosaminoglycans) in various body tissues owing to insufficient amounts of the enzyme galactosamine sulphate (type A) and galactosidase (type B) to break them down. A deficiency of either enzyme leads to the accumulation of mucopolysaccharides in the body, abnormal skeletal development and additional symptoms. The symptoms are variable, with types A and B being clinically indistinguishable. The incidence of MPS IV (Morquio-Brailsford syndrome, more commonly known as Morquio syndrome) varies with geographic location - about 1 in every 75000 births in Northern Ireland for Morquio syndrome type A, for example. Symptoms are usually noticed between ages 1 and 3 years. A family history of the syndrome raises the risk for the condition. It was first described, simultaneously and independently, in 1929, by Luis Morquio in Montevideo, Uruguay, and James Brailsford in Birmingham, England. ${ }^{1,2}$ They both recognised the occurrence of corneal clouding, aortic valve disease and urinary excretion of keratan sulphate. Morquio observed the disorder in 4 siblings in a family of Swedish extraction.

Patients with Morquio syndrome usually can be clinically distinguished from patients with other forms of MPS in that their intelligence is unimpaired, in contrast with other forms of MPS. Husler coined the term dysostosis multiplex to describe the skeletal findings.
The radiological features in our patient were consistent with dysostosis multiplex. Radiographic features include oval vertebral bodies with anterior beaking and unossified femoral heads with proximal femoral valgus deformities and broad, flat (wine-glass shape) ilia.

\section{Case report}

A boy presented with complaints of short stature, limping and spinal deformity. Physical examination showed pectus carinatum, short neck, knock knees, kyphosis, projecting jaw, broad mouth and flat feet. Hypermobility of all the joints was noted. The limbs were normal in length. There was marked hepatosplenomegaly with coarse facies and abnormal gait. Intelligence and development was appropriate for age. His slit-lamp examination, ECG and audiometry were completely normal .After radiological evaluation, he was confirmed as a case of Morquio syndrome. X-ray of the skull showed frontal bossing with J-shaped sella (Figs 1B and 1C). There was odontoid hypoplasia (one of the most critical feature of Morquio syndrome) along with atlantoaxial subluxation in radiographs of the cervical spine performed in flexion and extension views (Figs 1C and 1D). There were vertebra plana, or flattened vertebral bodies, with kyphoscoliosis and central beaking (Figs 1 and 2). This can be distinguished from the ovoid vertebral bodies of Hurler's syndrome and other mucopolysaccharidoses. The central anterior beaking of Morquio's syndrome also differs from the anteroinferior beaking of Hurler's syndrome and other mucopolysaccharidoses. Broadening of the anterior portion of the ribs (tongue shaped) was observed (Fig. 2). AP radiography of both hands with wrist revealed conical (bullet-shaped) proximal bases of 2 - 5 metacarpals with normal construction of metacarpal shafts. The ossified carpal bones were small and reduced in number for the patient age. The planes of both the distal ulnar and radial growth plates were slanted towards each other (Fig. 3). The pelvic film showed flared ilium laterally with inferior constriction (wine-glass shape) (Fig. 4). Frontal view of the femurs revealed enlarged acetabular cavities with rough margins, and poorly formed femoral epiphyses and widened femoral necks with coxa valga (Fig. 4). X-rays of both knees in AP view and legs with feet in AP and lateral views showed genu valgus, metaphyseal expansion of long bones, and tapering of the proximal phalanges respectively (Fig. 5). All these typical radiological features with specific laboratory findings confirmed this case as the rare metabolic syndrome of Morquio syndrome. 


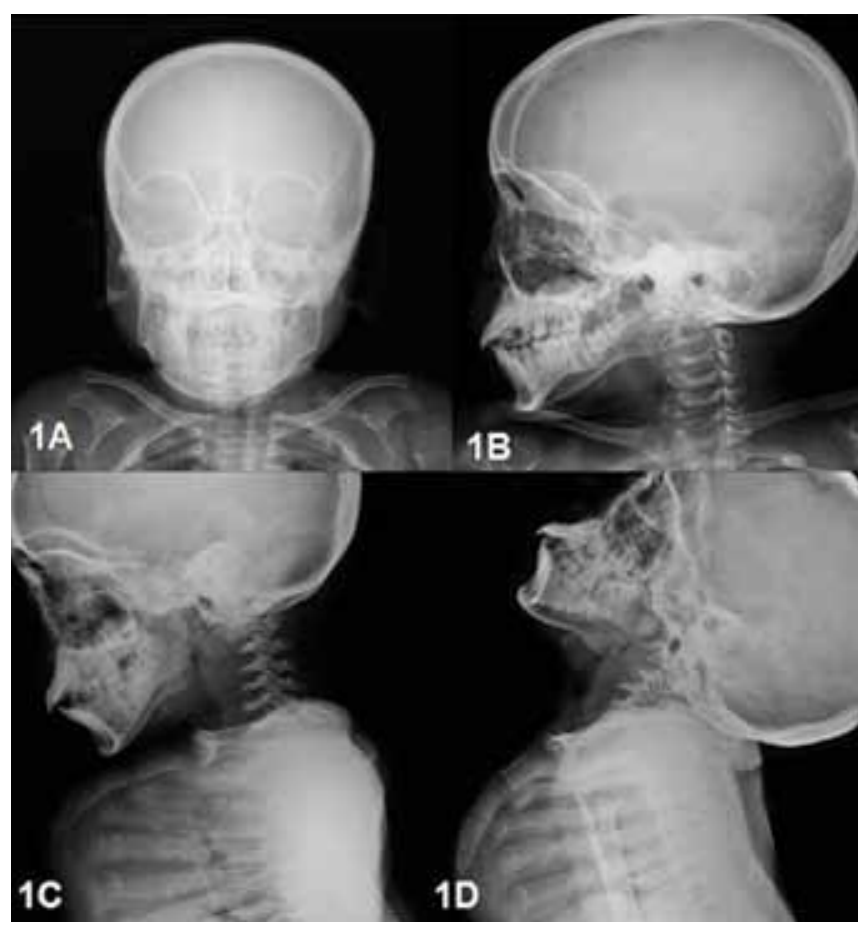

Fig. 1. X-ray of skull; $A P$ and lateral view (1A and $1 B$ ) and cervical spine in flexion and extension position (1C and 1D) showing frontal bossing and $a$ J-shaped sella. Atlanto-axial subluxation is seen in the cervical spine, and multi-level vertebra plana (flattened vertebrae).

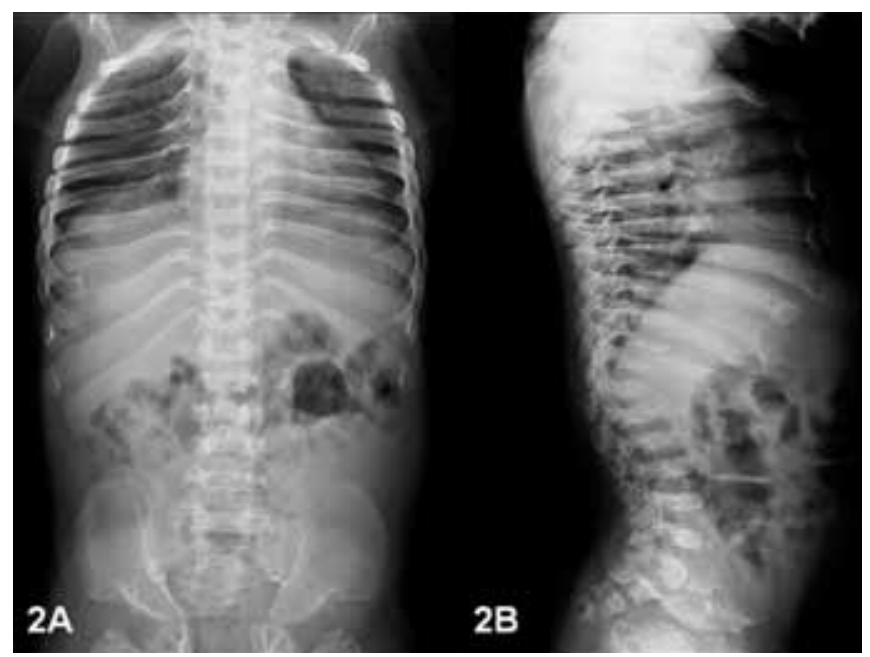

Fig. 2. X-ray of dorso-lumbar spine in $A P$ and lateral view ( $2 A$ and $2 B$ ) showing kyphosis with multi-level vertebra plana with central anterior beaking. Ribs show broadening of the anterior portion (tongue shape).

\section{Discussion}

Morquio's syndrome is a genetic disorder caused by the lack of the enzyme responsible for the breakdown of the mucopolysaccharide keratin sulphate. The resulting build-up of keratin sulphate causes a specific pattern of abnormalities. Affected children have normal intelligence and usually survive well into adulthood. Two forms are recognised: Type A, a deficiency of the enzyme galactosamine- 6 sulphatase; and Type B, a deficiency of the enzyme beta-galactosidase.

A genetic defect has been identified in the $\mathrm{N}$-acetyl-galactosamine-6sulphate sulphatase (GALNS gene) in Morquio's syndrome type IVA or

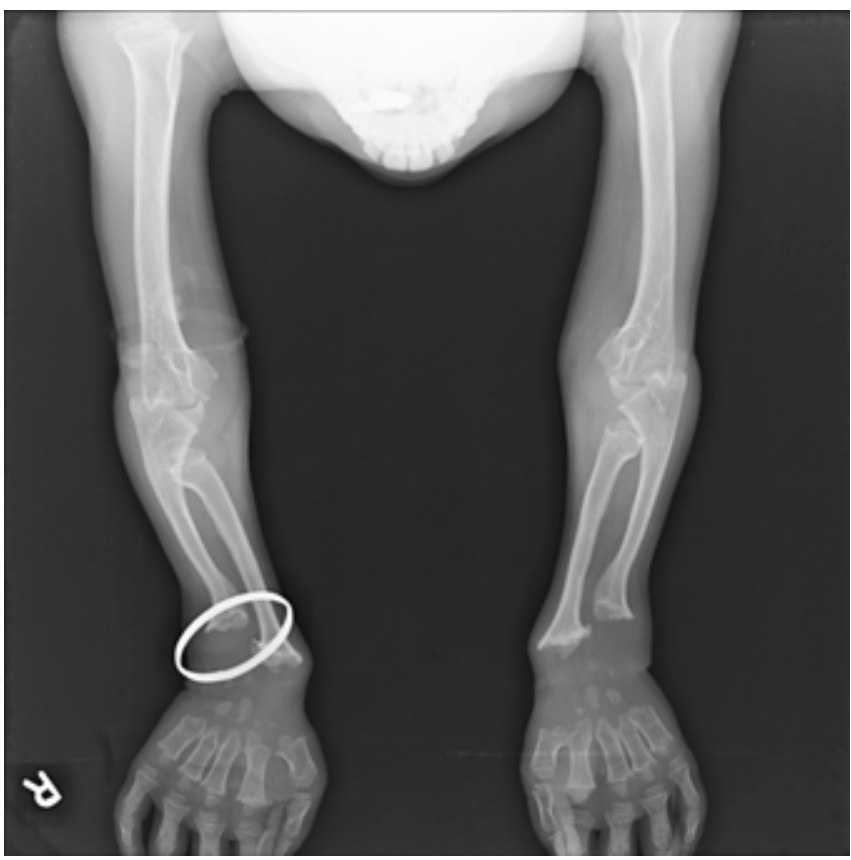

Fig. 3. X-ray of hands and wrists in AP view showng metaphyseal expansion of long bones with cortical thinning, conical (bullet-shaped) proximal bases of 2 - 5 metacarpals with normal construction of metacarpal shafts and tapering of the proximal phalanges. The ossified carpal bones are small and reduced in number for the patient's age. The planes of both the distal ulnar and radial growth plates are slanted towards each other.

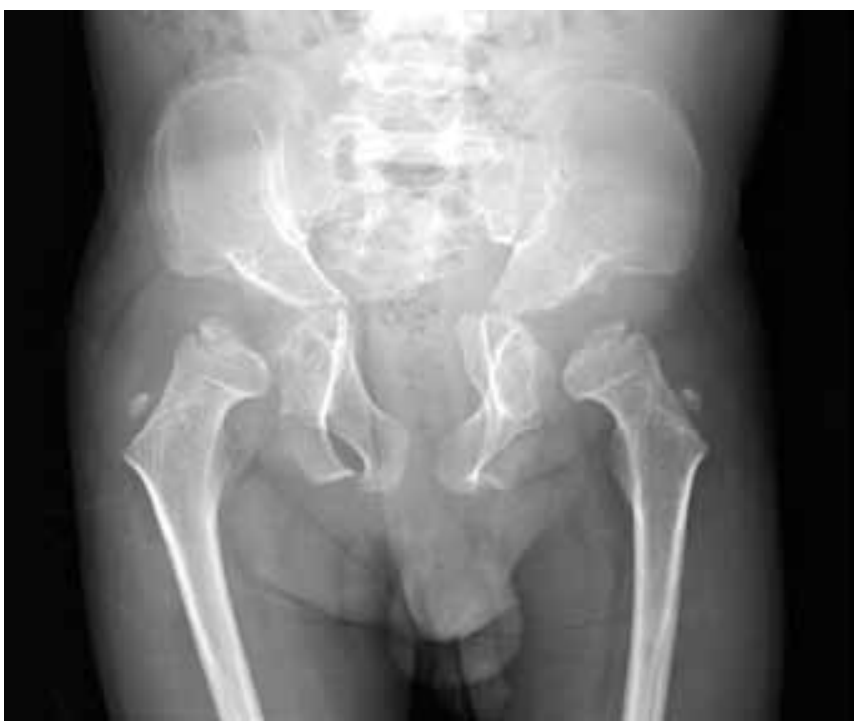

Fig. 4. X-ray of the pelvis and both femurs in AP view shows flared iliac bones laterally with inferior constriction (wine-glass shape). Enlargement of both acetabular cavities is seen with rough margins, and poorly formed femoral epiphyses and widened femoral necks with coxa valga.

beta-galactosidase (GLB1 gene) in Morquio's syndrome type IVB. The catabolism of chondroitin 6-sulphate is also affected by the GALNS gene defect. $^{3}$ The metabolism of heparan and dermatan sulphate is normal in Morquio syndrome, which is why patients with Morquio syndrome do not have mental retardation. Patients with Morquio's syndrome appear healthy at birth. Marked dwarfism, abnormal curvature of the spine (kyphoscoliosis), and decreased tone and weakness are prominent early childhood presentations. Also seen are coarse facies, short nose, 


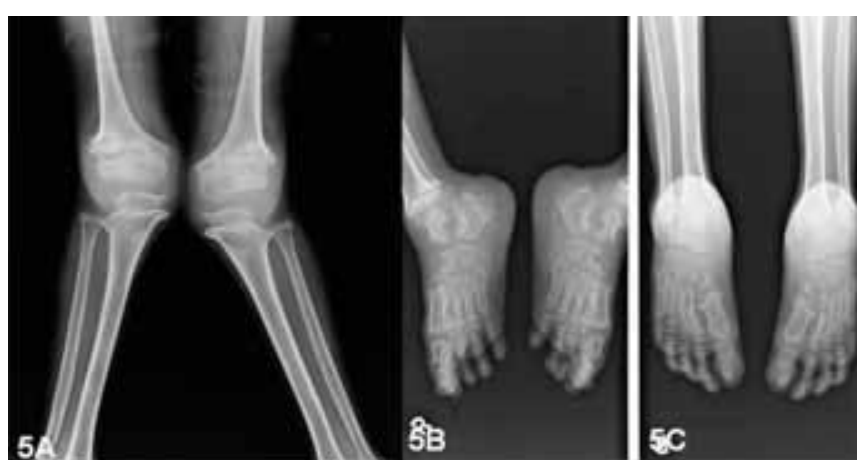

Fig. 5. X-ray of both knees in $A P$ view (5A) and leg with foot in $A P(5 B)$ and lateral view (5C) showing genu valgus, metaphyseal expansion of long bones, and tapering of the proximal phalanges respectively.

broad mouth, and widely spaced teeth with thinned enamel. The patient may have a waddling gait. Pectus carinatum (horizontal and protuberant sternum) and a shortened neck with clouding of the cornea, ligamentous laxity and joint stiffness are also seen. Mental capacity is generally unimpaired.

Examination and testing may reveal short stature (flat vertebrae cause a short trunk), short neck, kyphosis or scoliosis with pectus carinatum (pigeon chest) and, at the cervical spine, odontoid hypoplasia; atlantoaxial instability may be associated with myelopathy with gradual loss of walking ability. ${ }^{4}$ Laxity of joints, dysostosis multiplex, dysplastic hips, unstable knees, large elbows and wrists, and flat feet are other skeletal features. There may be cardiac abnormalities with enlarged liver and spleen. Investigations include urine spot tests which are readily available to screen for mucopolysaccharides but are associated with false-positive and false-negative results. Heparan, keratan and dermatan sulphate can be distinguished by electrophoresis techniques to differentiate between the mucopolysaccharidoses. The diagnosis is confirmed by direct enzyme assay of leucocytes, or fibroblast enzyme activity can be measured in amniocytes or chorionic villi.

Roentgenographic findings have been reviewed by Langer and Carry. ${ }^{5}$ Compared with other forms of MPS, Morquio syndrome tends to have greater skeletal manifestations and spine involvement such as scoliosis, kyphosis, hyperlordosis, severe gibbus, flaring of the lower ribs as well as platyspondyly, pectus carinatum metacarpals, and small carpal bones (often with some absent). Characteristic findings included hypoplasia of odontoid peg, universal platyspondyly gibbus, and kyphosis of the dorsal region with widening of disc spaces. Also characteristic are a long pelvis with narrowing at the acetabulae, pubic symphysis widening and flaring of the ilia. There may be shortening of the metacarpals, and inclination of the distal portions of the radius and ulna toward each other can also be seen (as in our case).

Complications develop later and include breathing problems, cardiac problems, spinal cord damage leading to possible paralysis, vision problems, walking problems related to abnormal curvature of the spine, and other bony problems. Most patients survive into their third or fourth decades. Treatment is currently only palliative. Possible future treatments include enzyme replacement, gene therapy and allogenic bone marrow transplantation. ${ }^{6}$

1. Morquio L. Surune forme de dystrophie osseuse familiale. Bull Soc Pediatr (Paris) 1929;27:145-152

2. Brailsford JF. Chondro-osteo-dystrophy: Roentgenographic and clinical features of child with dislocation of vertebrae. Am J Surg 1929;7:404-410.

3. Fukuda S, Tomatsu S, Masue M, et al.: Mucopolysaccharidosis type IVA. N-acetylgalactosamine-6-sulfate sulfatase exonic point mutations in classical Morquio and mild cases. J Clin Invest 1992;90:1049-1053.

4. Montano AM, Tomatsu S, Gottesman GS, Smith M, Orii T. International Morquio A Registry: clinical manifestation and natural course of Morquio A disease. J Inherit Meta Dis 2007;30(2):165-174.

5. Langer LO, Carey LS. The roentgenographic features of the KS mucopolysaccharidosis of Morquio. Am J Roentgenol 1966:97:1-20.

6. Tomatsu S, Montano AM, Ohashi A, et al. Enzyme replacement therapy in a murine model of Morquio A syndrome. Hum Mol Genet 2008;17(6):815-824. 